\title{
Staging of hepatocellular carcinoma
}

\author{
Sedat Karademir \\ Department of General Surgery, Section of HPB Surgery and Organ Transplantation, Ankara Guven Hospital, Ankara 06540, Turkey. \\ Correspondence to: Prof. Sedat Karademir, Department of General Surgery, Section of HPB Surgery and Organ Transplantation, Ankara \\ Guven Hospital, Ankara 06540, Turkey. E-mail: sedatkarademir@gmail.com
}

How to cite this article: Karademir S. Staging of hepatocellular carcinoma. Hepatoma Res 2018;4:58.

http://dx.doi.org/10.20517/2394-5079.2018.40

Received: 16 Apr 2018 First Decision: 29 Jun 2018 Revised: 13 Aug 2018 Accepted: 22 Aug 2018 Published: 26 Sep 2018

Science Editor: Guang-Wen Cao Copy Editor: Yuan-Li Wang Production Editor: Zhong-Yu Guo

\begin{abstract}
Hepatocellular carcinoma (HCC) is the third leading cause of cancer deaths in the world. In contrast to other cancers, survival of patients with HCC is determined by the extent of the tumor in addition to underlying liver disease and its functional reserve. From risk factors to management, HCC reveals a considerable geographic and institutional variation throughout the world. Although many staging and/or scoring systems have been proposed, each prognostic system has several benefits and limitations on its own. Therefore, there is currently no globally accepted system for HCC due to the extreme heterogeneity of the disease. In this review, currently available staging systems for assessing the prognosis of HCC, their uses, limitations, and future prospects are revisited.
\end{abstract}

Keywords: Hepatocellular cancer, staging and scoring systems, risk factors, survival

\section{INTRODUCTION}

Cancer staging systems are important for identification of appropriate therapies and prediction of prognosis for individual patients. Staging in cancer also helps to create a common language in clinical investigations and research $^{[1]}$. Since last 2 decades, several staging systems have been proposed for hepatocellular carcinoma (HCC). However, no single system has been universally accepted. Each staging system reflects the features of its own patient population that has been studied on. From risk factors to the treatment given for HCC, considerable geographic and institutional variations exist worldwide. In addition to heterogeneity of the tumor, in each country, availability of surveillance programs, quality of medical technology and accessibility to treatment may influence the prognosis of HCC patients. A staging system which is found to be useful in Western countries may not be similarly suitable for Eastern population. However, external validation of a proposed system for different patient groups worldwide is crucial to reach a common guideline for the management of HCC. So, the

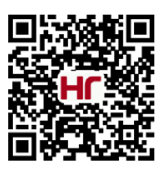


Table 1. Components of HCC scores and staging systems published in the recent years

\begin{tabular}{|c|c|c|c|c|c|c|c|c|}
\hline \multirow[b]{2}{*}{ Staging systems } & \multirow{2}{*}{$\begin{array}{l}\text { Liver functional } \\
\text { reserve }\end{array}$} & \multirow{2}{*}{$\begin{array}{c}\text { Performance } \\
\text { status, symptoms }\end{array}$} & \multirow[b]{2}{*}{ AFP } & \multicolumn{4}{|c|}{ Tumor status } & \multirow[b]{2}{*}{ Other } \\
\hline & & & & Number & Size & $\begin{array}{l}\text { Vascular } \\
\text { invasion }\end{array}$ & Metastasis & \\
\hline Okuda $(1985)^{[6]}$ & $\begin{array}{l}\text { Ascites, albumin, } \\
\text { bilirubin }\end{array}$ & & & & Yes & & & \\
\hline CLIP $(1998)^{[7]}$ & Child-Pugh score & & Yes & Yes & Yes & Yes & & \\
\hline French $(1999)^{[8]}$ & Bilirubin & Karnofsky scale & Yes & & & Yes & & Alk-P, PVT \\
\hline $\operatorname{BCLC}(1999)^{[3]}$ & Child-Pugh score & EGOS PST & & Yes & Yes & Yes & Yes & \\
\hline AJCC TNM-7 (2010) & & & & Yes & Yes & Yes & Yes & \\
\hline CUPI $(2002)^{[9]}$ & Ascites, bilirubin & Symptoms & Yes & Yes & Yes & Yes & Yes & Alk-P \\
\hline JIS $(2003)^{[10]}$ & Child-Pugh score & & & Yes & Yes & Yes & Yes & \\
\hline m-JIS $(2006)^{[57]}$ & $\begin{array}{l}\text { +ICG-R15 (- } \\
\text { encepalopathy) }\end{array}$ & & & Yes & Yes & Yes & Yes & \\
\hline bm-JIS (2008) & Child-Pugh score & & Yes & Yes & Yes & Yes & Yes & AFP-L3, DCP \\
\hline Tokyo $(2005)^{[11]}$ & Albumin, bilirubin & & & Yes & Yes & & & \\
\hline BALAD $(2006)^{[60]}$ & Albumin, bilirubin & & Yes & & & & & AFP-L3, DCP \\
\hline $\operatorname{ALPCS}(2008)^{[48]}$ & Child-Pugh score & Symptoms & Yes & & Yes & & Yes & Alk-P, Urea, PVT \\
\hline $\operatorname{TIS}(2010)^{[63]}$ & Child-Pugh score & & Yes & Total tumo & volume & & & \\
\hline $\operatorname{MESIAH~}(2012)^{[12]}$ & MELD, albumin & Age & Yes & Yes & Yes & Yes & Yes & \\
\hline $\operatorname{HKLC}(2014)^{[4]}$ & Child-Pugh score & EGOS PST & No & Yes & Yes & Yes & Yes & \\
\hline ITA.LI.CA $(2016)^{[68]}$ & Child-Pugh score & EGOS PST & Yes & Yes & Yes & Yes & Yes & \\
\hline
\end{tabular}

HCC: hepatocellular carcinoma; CLIP: Cancer of the Liver Italian Program; BCLC: Barcelona Clinic Liver Cancer; AJCC: American Joint Committee of Cancer; TNM: tumor-node-metastasis; CUPI: Chinese University Prognostic Index; JIS: Japan Integrated Staging; m-JIS: modified JIS; bm-JIS: biomarker combined JIS; BALAD: bilirubin-albumin-AFPL3-AFP-DCP; ALPCS: advanced liver cancer prognostic system; TIS: Taipei Integrated Scoring System; MESIAH: model to estimate survival in ambulatory HCC; HKLC: Hong Kong Liver Cancer; AFP: alpha-fetoprotein; AFP-L3: AFP-Lens culinaris agglutinin-reactive; DCP: des-gamma-carboxy prothrombin; EGOS PST: Eastern Cooperative Oncology Group performance status; ICG-R15: indocyanine green clearance; PVT: portal vein thrombosis

search for a simple, reliable, reproducible and comprehensive staging system continues.

Most HCCs develop upon chronic diseases of the liver, mainly B or C viral hepatitis. Due to the underlying liver disease, prognosis of HCC depends not only on extend of the tumor but also on functional reserve of liver, overall health status of the patient and the treatment given for $\mathrm{HCC}^{[2]}$. For an accurate prognostication of HCC, parameters which look at all these aspects of prognosis must be included in staging process. In addressing interrelationship of prognostic factors in HCC, several staging systems have been developed but only a few have been widely used and validated.

To date, various parameters have been studied to be of prognostic usefulness in patients with HCC. Parameters based on systematic reviews of the literature and/or expert opinions ${ }^{[3-5]}$ as well as variables that were significant in multivariable Cox survival analyses ${ }^{[6-12]}$ were incorporated in these staging/ scoring systems. Besides the simple patient related demographic data such as age and gender, many other specific biochemical and clinical variables of liver function, tumor burden and biology as well as agerelated clinical consequences and comorbidities have been included in regression analysis of the different studied populations worldwide ${ }^{[1]}$ [Table 1]. Several biomarkers have also been studied for their prognostic significance in patients with $\mathrm{HCC}^{[13]}$.

The treatment options for patients with HCC are expanding. Depending on the stage of the disease, surgical resection, percutaneous ablation, transarterial chemoembolization and transplantation are being performed either singly or as combination of various modalities. For patients with advanced disease, sorafenib, a multikinase inhibitor is also available. The choice of therapy is influenced by several factors including stage of tumor and severity of underlying liver dysfunction as well as availability of resources and of expertise. Thus, to reach a single staging system and treatment algorithm applicable to all patients with HCC seems to continue to be challenging. 
Table 2. Okuda scoring system ${ }^{[6]}$

\begin{tabular}{ll}
\hline Parameters of advance disease & \\
\hline Tumor involving $>50 \%$ of the liver & \\
Ascites & \\
Albumin $<3 \mathrm{~g} / \mathrm{dL}$ & \\
Bilirubin $>3 \mathrm{mg} / \mathrm{dL}$ & No positive parameter \\
Stage I & 1 or 2 positive parameter(s) \\
Stage II & 3 or 4 positive parameters \\
Stage III & \\
\hline
\end{tabular}

\section{CURRENTLY AVAILABLE STAGING SYSTEMS FOR HCC}

\section{Okuda score}

Okuda staging system was proposed in 1985 based on a study of 850 HCC patients ${ }^{[6]}$. This system is the first to combine tumor size ( $\leq$ or $>50 \%$ of the entire liver) with the variables of liver function such as ascites (presence and absence), serum albumin ( $\leq$ or $>3.0 \mathrm{~g} / \mathrm{dL}$ ) and bilirubin levels $(\leq$ or $>3.0 \mathrm{mg} / \mathrm{dL}$ ). Based on these variables, patients are classified into three stages (I: not advanced; II: moderately advanced; III: very advanced) with different outcomes [Table 2]. Okuda staging system was accepted and widely used as an improved classification system for HCC. However, at the time of its introduction, most HCC cases were diagnosed in the advanced stage (18.5\% had surgery). It hardly differentiates the less advanced patients. Therefore, Okuda system is not suitable for the majority of current HCC patients, who are often diagnosed at an early, asymptomatic stage of the disease with possible indication for today's therapeutic modalities. Also, there are major concerns about this system. Considering recent advances in imaging techniques, the only tumor related variable, tumor size ( $\leq$ or $>50 \%$ of the entire liver) is defined somewhat arbitrarily. It does not include vascular invasion, multicentricity or extrahepatic spread of tumor which definitely affect patient outcomes ${ }^{[14]}$. Instead of differentiating early from advance stages, it was found to be useful mainly to identify end-stage patients (stage III), that should be excluded from therapeutic trials due to their poor prognosis. When compared with modern staging systems, it has been shown to have lower predictive capacity ${ }^{[15-19]}$. Despite these shortcomings, the Okuda staging system has remained a widely accepted and simple classification system for HCC.

\section{Cancer of the Liver Italian Program score}

The Cancer of the Liver Italian Program (CLIP) score was proposed by an Italian group in 1998 based on a retrospective analysis of 435 HCC patients treated at 16 Italian institutions ${ }^{[7]}$. Of these, only $12(2.8 \%)$ had surgery and $247(56.8 \%)$ underwent locoregional therapy. CLIP was designed to overcome the deficiencies of the tumor-node-metastasis (TNM) system. It takes into account the Child-Pugh status of the patient with tumor characteristics including tumor morphology and extension, the portal vein thrombosis and levels of alfa-fetoprotein (AFP) assign a score $(0,1,2)$ to each variable [Table 3]. Patients are classified into seven groups according to the sum of these scores (0-6). CLIP is easy to calculate, well correlated with survival. CLIP-0 patient has a better prognosis in comparison to one with CLIP-6 (42.5 mo vs. 1.0 mo of median survival). However, in this system, information regarding underlying liver diseases, performance status and extrahepatic metastasis which affect the outcomes were lacking. Additionally, it does not offer any appropriate therapy for HCC patients.

This scoring system was validated prospectively in 196 HCC patients and showed greater predictive power than Okuda staging system ${ }^{[20]}$. Although, the CLIP score was developed using an appropriate method and has been externally validated in several (Canadian, Italian and Japanese) $\operatorname{cohorts}^{[18-21]}$, this score has some limitations when applied to patients with the early stage of HCC. In countries like Japan, where many smaller tumors are detected based on the established screening system for HCC, the CLIP score cannot effectively identify early-stage patients who can benefit from radical treatment. 
Table 3. CLIP scoring system ${ }^{[7]}$

\begin{tabular}{ll}
\hline & Scores \\
\hline Child-Pugh stage & 0 \\
A & 1 \\
B & 2 \\
C & \\
Tumor morphology & 0 \\
$\quad$ Uninodular and extension $\leq 50 \%$ & 1 \\
Multinodular and extension $\leq 50 \%$ & 2 \\
Massive or extension $>50 \%$ & \\
Alpha-fetoprotein (ng/dL) & 0 \\
$<400$ & 1 \\
$\geq 400$ & \\
Portal vein thrombosis & 0 \\
No & 1 \\
Yes & \\
\hline
\end{tabular}

CLIP: Cancer of the Liver Italian Program

Table 4. French scoring system ${ }^{[8]}$

\begin{tabular}{llccc}
\hline & \multicolumn{4}{c}{ Scores } \\
\cline { 2 - 5 } & $\mathbf{0}$ & $\mathbf{1}$ & $\mathbf{2}$ & $\mathbf{3}$ \\
\hline Karnofsky index $(\%)$ & $\geq 80$ & & & $<80$ \\
Serum bilirubin $(\mu \mathrm{mol} / \mathrm{L})$ & $<50$ & & & $\geq 50$ \\
Serum alkaline phosphatase $(\mathrm{ULN})$ & $<2$ & & $>2$ & \\
Serum alpha-fetoprotein $(\mu \mathrm{g} / \mathrm{L})$ & $<35$ & & & \\
Portal obstruction $($ sonography) & No & Yes & & \\
\hline
\end{tabular}

ULN: upper limit normal

In different studies, nearly $80 \%$ of the patient population is classified as having a CLIP score of 0-2 which shows its poor stratification ability ${ }^{[1,22,23]}$. One possible reason may be the definition of tumor extension (less or more than $50 \%$ of total liver volume) which is somewhat subjective and may compromise the reliability of CLIP in predicting patient outcomes ${ }^{[24-27]}$. Still, CLIP is recently ranked first for its ability to predict $\operatorname{survival}^{[22]}$.

\section{GRETCH Score}

GRoupe d'Etude et de Traitement du Carcinoma Hépatocellulaire (GRETCH) system was proposed by the French group Goupe d'Etude et de in $1999^{[8]}$. This system was constructed with the analysis of 761 HCC patients treated at 24 centers. The group has created a score quite similar to the CLIP aiming at a simple classification that would predict survival. Unlike CLIP, GRETCH further includes performance status but lacks tumor morphology information. GRETCH staging divides the patients into three risk groups (A, B, C) on the basis of performance status, serum bilirubin, serum alkaline phosphatase (ALP), serum AFP and portal vein obstruction on ultrasound [Table 4]. The overall survival (OS) differs markedly for the three groups, with a one-year survival rate in group A (low risk to death) of $72 \%$, compared to $34 \%$ in group B (intermediate risk of death) and $7 \%$ in group C (high risk of death). The strength of this system is that it is based on baseline characteristics that are routinely available at diagnosis and the scores allocated to the respective predictive factors are based on the estimated Cox regression coefficient. However, in this study, $53 \%$ of HCC patients did not receive any specific therapy, while only $7.4 \%$ underwent surgical resection. Therefore, this score may not be suitable for predicting the survival of HCC patients who undergo surgical resection. In addition, this cohort mostly included patients at advanced stages. A recent comparison with other staging systems has shown that it has limited prognostic capacity in patients with early HCC $^{[15]}$. 


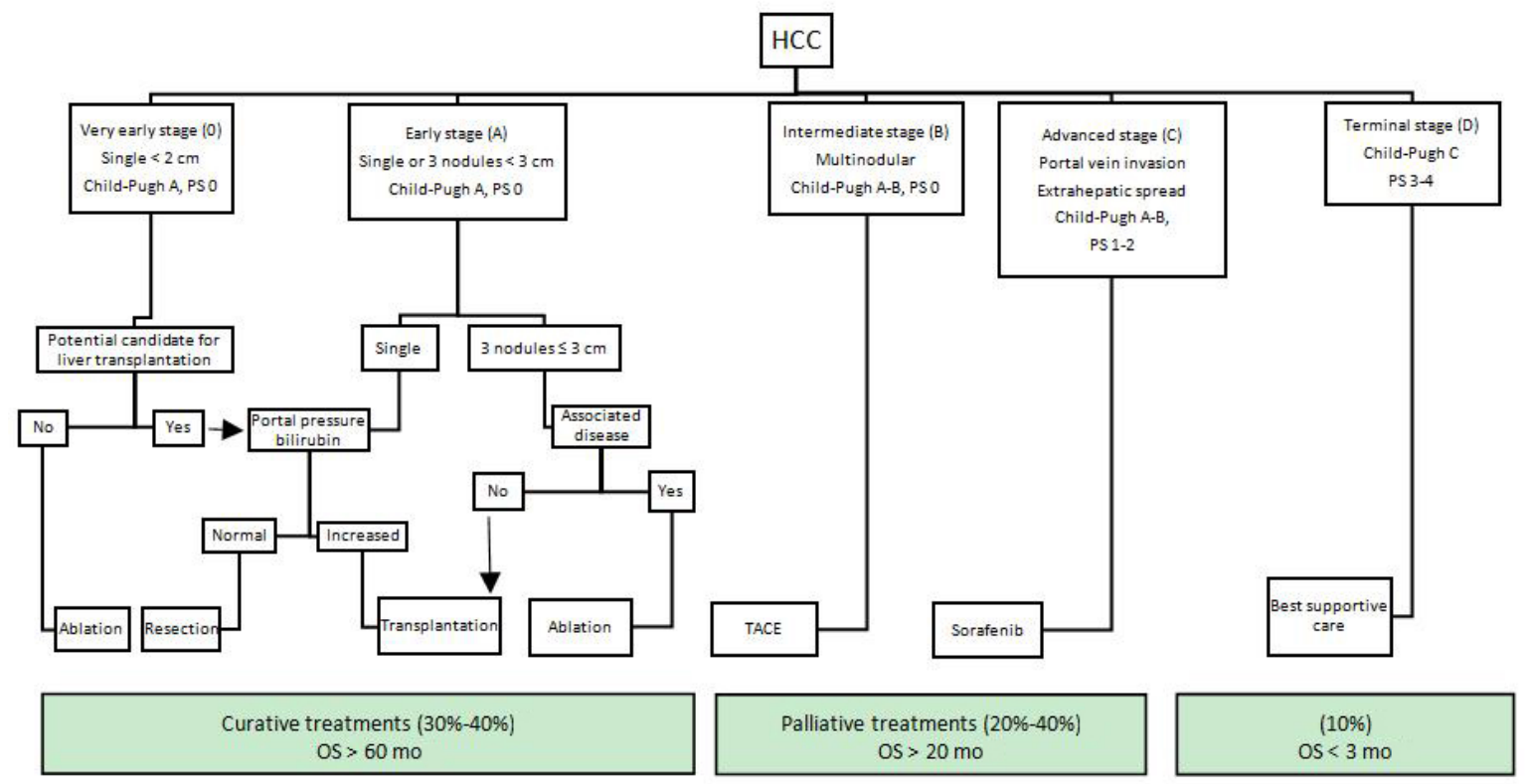

Figure 1. $B C L C$ staging system. PS: performance status; OS:overall survival; BCLC:Barcelona Clinic Liver Cancer

Another issue regarding GRETCH score is use of ultrasound in face of recent advances in imaging. More informative techniques rather than ultrasound may be used to evaluate portal venous anatomy.

\section{Barcelona Clinic Liver Cancer classification}

The Barcelona Clinic Liver Cancer (BCLC) classification for HCC was proposed by Llovet et al ${ }^{[3]}$ in 1999. The BCLC staging system is developed from the results obtained in the setting of several cohort studies and $\mathrm{RCTs}^{[1,3,28]}$. As it is not based on identification of prognostic factors from a regression analysis, BCLC is not able to predict the mortality in HCC patients ${ }^{[26]}$. The notable feature of BCLC is being the first system which recommends evidence-based clinical treatment for each patient at different stages [Figure 1]. Its treatment algorithm has also been recognized as a guideline by renown societies (the American Association for the Study of Liver Diseases, the American Gastroenterological Association and European Association for the Study of the Liver) for management of $\mathrm{HCC}^{[29,30]}$.

BCLC includes predictors of prognosis in HCC patients including tumor extension, liver functional reserve and overall physical status (PS). Tumor extension contains the number of tumors, tumor size and presence of portal vein invasion or extrahepatic metastasis. Child-Pugh grade is replaced for liver functional reserve and the PS is determined by corresponding Eastern Cooperative Oncology Group (ECOG) performance status $^{[31]}$. Patients are subsequently assigned to five categories (0, A, B, C and D) based on these variables: stage 0 (very early stage) describes patients with well-preserved liver function (Child-Pugh A), one asymptomatic tumor $<2 \mathrm{~cm}$ and no vascular invasion or satellites. Stage A (early stage) covers Child-Pugh A or B patients with one tumor of any size or $2-3$ tumors all $<3 \mathrm{~cm}$. Stage B (intermediate-stage) defines patients with ChildPugh A or B status, multiple tumors without vascular invasion or extrahepatic metastasis. Child- Pugh A or B patients with vascular invasion or extrahepatic metastasis of tumor and in relatively good PS (1-2) are assigned to stage C (advanced stage). Finally, stage D (terminal stage) corresponds to patients with ChildPugh C status in any tumor stage and poor PS $(>2)^{[3,32]}$.

Overall, the BCLC staging system identifies early, intermediate, advanced, and end stage HCC patients who may benefit from curative therapies, palliative treatments or best supportive care. Curative treatment options such as surgical resection, liver transplantation and ablation are recommended for patients with 
early stage HCC (stages 0 and A) (median OS > 60 mo). For stage B patients, palliation with transarterial chemoembolization (TACE) is recommended with median OS of 20 months. Sorafenib, multikinase inhibitor, was added to treatment repertoire in 2008 for patients with advanced disease (stage C) (median $\mathrm{OS}=11 \mathrm{mo}$ ). And, for patients at terminal stage with life expectancy of $<3$ months, best supportive care is recommended.

Compared to Okuda and CLIP systems, early stage HCC is defined in more details (number and size of nodules, the associated comorbidities and the portal vein pressure) in BCLC which makes it more suitable to select early stage patients who could benefit from curative therapies ${ }^{[1,33]}$. However, the BCLC has shown to have lower prognostic ability than CLIP score regarding advance HCC $^{[34,35]}$.

BCLC has been externally validated in several Western countries where main etiologies are hepatitis $C$ virus $(\mathrm{HCV})$ infection and alcohol abuse ${ }^{[2]}$ and are found to have a better ability to predict survival than most other staging systems ${ }^{[23,36]}$. Although BCLC has been widely used in Western countries, many Asian experts find its treatment modalities to be too conservative. In contrast to BCLC, Asian guidelines indicate surgical resection and TACE for more advanced tumors. Even then, some studies by Asian groups proved BCLC to be a superior staging system ${ }^{[37]}$.

Despite its popularity, several studies have shown that BCLC staging system has some limitations. These are mainly related to the heterogeneity of BCLC stages B and C patients in respect to tumor burden and liver function ${ }^{[38]}$. For example, patients with multinodular disease without vascular invasion are assigned to intermediate stage (BCLC B) and only a single therapeutic option, TACE is offered. However, resectability of multifocal HCC is closely related to location of tumors. A patient with multiple small tumors confined to the same lobe may still be considered as a good candidate for resection, instead of transarterial chemoembolization. Additionally, tumors with portal invasion (BCLC C) are recommended to be treated only with sorafenib ${ }^{[29]}$. For these patients, there are studies which suggest extending the indication for surgery ${ }^{[39-41]}$ or chemoembolization ${ }^{[42,43]}$. Even for a Child-Pugh C patient with HCC within the Milan criteria, the possibility of liver transplantation may be considered. On the other end, in BCLC 0 and A patients with early stage HCC, a single liver tumor is resected only in absence of portal hypertension where it might not affect survival in many resected patients. In current practice, sequential or combined treatments are highly preferred in the multidisiplinary management of HCC (TACE followed by resection or LT, TACE + RF or sorafenib). Under these circumstances, BCLC's one-to-one correspondence treatment recommendations for each stage may not be suitable for use in actual clinical practice ${ }^{[26,44]}$. Another critic on BCLC is regarding the controversial prognostic role of variable ECOG PS which is somewhat subjective and may affected by liver function and cancer symptoms ${ }^{[45]}$.

The BCLC has shown to have lower prognostic ability than CLIP score regarding advance HCC ${ }^{[36,46,47]}$. A new score, advanced liver cancer prognostic system (ALCPS) was proposed by Yau et al. ${ }^{[4]}$ aiming at improving patients selection but found to be too complex for daily clinical practice as it includes eleven variables with different coefficient as in the Chinese University Prognostic Index (CUPI) score ${ }^{[9]}$.

\section{TNM (AJCC)}

The TNM classification was developed by the American Joint Committee on Cancer (AJCC) and International Union for Cancer Control (UICC) and has been updated regularly since the first edition was published in 1977. This system is successfully used by oncologists in several fields. However, the classical staging system based on TNM is not used for HCC. It assesses the extension of the primary tumor, lymph node involvement and extrahepatic metastasis but does not include any measurements of liver function or the health status of the patient. Because of this it has often been used in combination with other criteria such as the Child-Pugh classification or included in other grading systems such as the CLIP score. TNM staging 
Table 5. Simplified staging system (Vauthey et al. ${ }^{[51]}$-TNM 6th edition)

\begin{tabular}{ll}
\hline ST1 & Single tumur without vascular invasion \\
sT2 & Single tumour with vascular invasion or multiple tumours, none $>5 \mathrm{~cm}$ \\
sT3 & Multiple tumours, any $>5 \mathrm{~cm}$ or tumour(s) involving major branch of hepatic vein(s) \\
FO & Grade $0-4$ fibrosis (no fibrosis to moderate fibrosis) \\
F1 & Grade 5-6 (severe fibrosis or cirrhosis) \\
Stage I & ST1 NO MO \\
Stage II & ST2 NO MO \\
Stage IIIA & ST3 NO MO \\
Stage IIIB & Any ST N1 MO \\
Stage IV & Any ST any N M1 \\
\hline
\end{tabular}

s: simplified; TNM: tumor-node-metastasis

system has been mostly tested in the surgical setting and showed poor prognostic prediction in early HCC patients undergoing either resection ${ }^{[49]}$ or transplantation ${ }^{[50]}$.

Vauthey et al. ${ }^{[51]}$ developed a simplified staging system for HCC in 2002 which was adopted as the TNM staging system of AJCC/UICC after minor changes (6th Edition) [Table 5]. It was derived from the finding of a cohort of 557 HCC patients who underwent surgical resection. The authors identified independent predictors of mortality (major vascular invasion, microvascular invasion, severe fibrosis/cirrhosis, multiple tumors and a tumor size greater than $5 \mathrm{~cm}$ ) using a multivariate analysis. Based on these variables, the AJCC $\mathrm{T}$ classification reclassified and a simplified stratification was proposed: $\mathrm{sT} 1$ : single tumor with no vascular invasion; sT2: single tumor with microvascular invasion or multiple tumors, none more than $>5 \mathrm{~cm}$ and sT3: multiple tumors, any $>5 \mathrm{~cm}$ or tumor(s) with major vascular invasion. The simplified staging system divides patients into 3 independent prognostic groups (5-year survival rates: stage I 55\%, stage II $37 \%$ and stage III 16\%). The new system may improve the stratification of resected tumors, even though it is controversial whether they will apply to nonsurgical patients. As TNM staging relies on detailed histopathologic examination which requires two fine-needle biopsies, this might be associated with risk of tumor seeding ${ }^{[52]}$.

The current AJCC/UICC 7th edition is a modification of the simplified staging system and has become widespread since $2010^{[5]}$. The major change between the 6th and the 7 th AJCC staging system is that the new system imposes heavier prognostic weight on major vascular invasion as a potential predictive factor for poor prognosis ${ }^{[53]}$. The main limitation of this staging system is that it fails to account for liver function whereas it is well known that prognosis of HCC patients also relies on features related to liver cirrhosis ${ }^{[4,50]}$.

\section{Japan Integrated Staging Score}

The Japan Integrated Staging Score (JIS Score) was proposed by Kudo et al. ${ }^{[10]}$ in 2003. It is derived from a cohort of 722 HCC patients treated at two Japanese institutions. The JIS score combines the ChildPugh grade with the Japanese TNM (Liver Cancer Study Group of Japan- LCSGJ) which is based on three parameters (vascular invasion, single $v s$. multiple nodules, diameter $\leq v \boldsymbol{s}$. $>20 \mathrm{~mm}$ ) to address the specific deficiency of LCSGJ for not having included liver function evaluation. Patients with a Child-Pugh grades $\mathrm{A}, \mathrm{B}$ and $\mathrm{C}$ status are allocated a score of 0,1 , and 2, respectively. Patients with the TNM stage by LCSGJ of stages I, II, III and IV are allocated to score of 0, 1, 2 and 3, respectively ${ }^{[54]}$ [Table 6]. Patients are subsequently classified into six groups (0-5) based on the sum of these scores. Statistically significant differences are observed between the survival curves for almost all JIS scores. The cumulative 10-year survival rates of the best prognostic groups in the CLIP staging system (CLIP score 0) and JIS staging system (JIS score 0) were $23 \%$ and $65 \%$, respectively. The authors concluded that the JIS score stratifies patients with early diagnosed HCC better than the CLIP score. The same group externally validated the JIS score in 4525 HCC patients treated at five Japanese institutions in $2004^{[55]}$. In a study of 1679 patient, the JIS score has been compared with the BCLC and CLIP and found to be superior in prognostic determination ${ }^{[56]}$. Since the JIS score was 


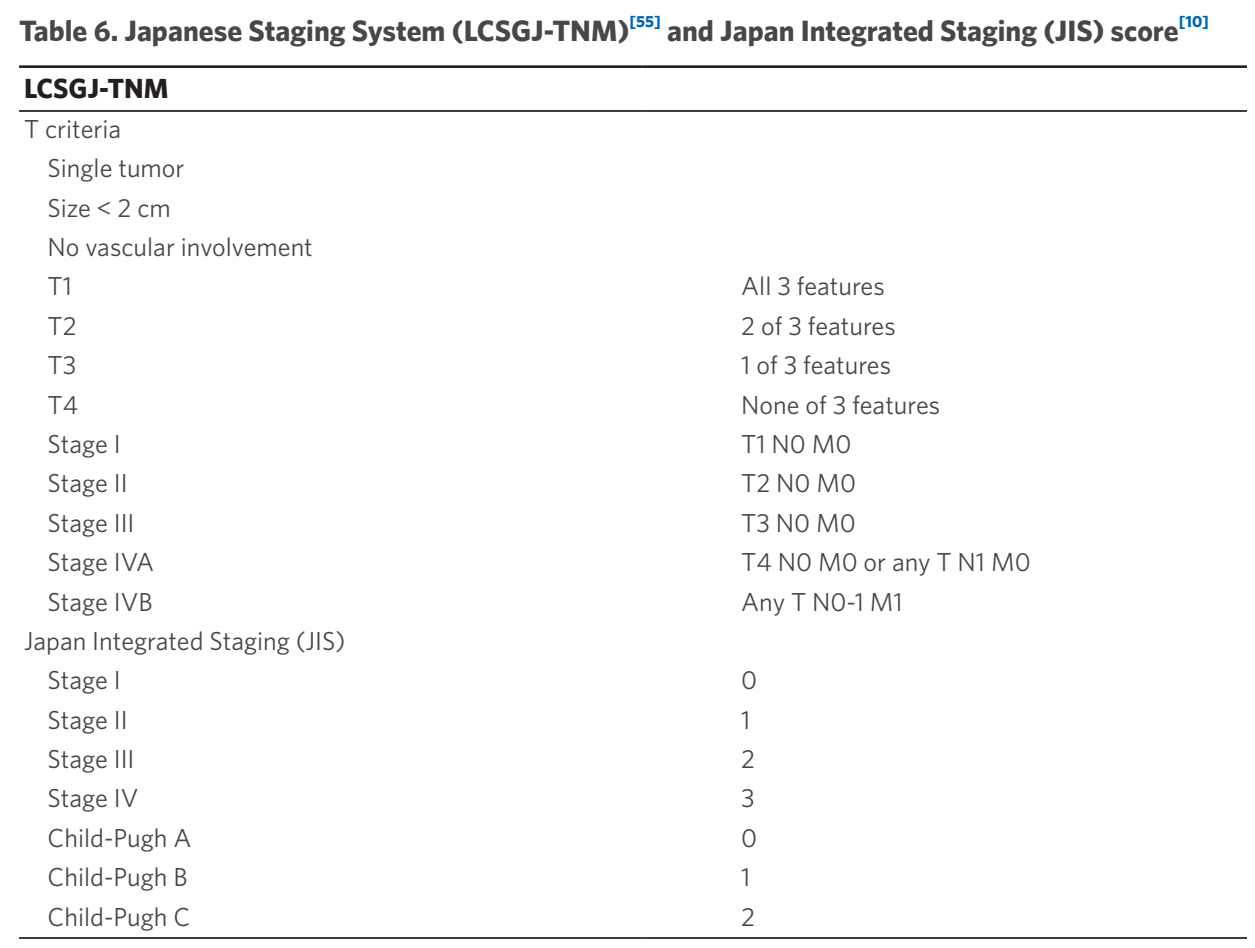

LCSGJ: Liver Cancer Study Group of Japan; TNM: tumor-node-metastasis, TNM: tumor-node-metastasis

developed based solely on Japanese HCC patients, prospective validation studies are required in Western population. The other limitations inherent in the LCSGJ staging system such as inaccurate weighting of size and vascular involvement as well as the lack of incorporation of microscopic pathology information remain.

This score was further improved a few years later with the modified-JIS in which the encephalopathy item is replaced by the indocyanine green clearance (ICG-R15), due to an early HCC screening in Japan and a preferred surgical orientation ${ }^{[57]}$. The substitution of ICG-R15 for encephalopathy in Child-Pugh grade might have reflected individual differences more accurately among patients who underwent hepatic resection, because none of the patients in the present study had any encephalopathy before operation ${ }^{[57]}$.

JIS has been recently refined as biomarker combined JIS (bm-JIS) by including AFP, AFP-Lens culinaris agglutinin-reactive (AFP-L3) and des-gamma-carboxy prothrombin (DCP) which allowed better survival predictions ${ }^{[58]}$. However, two of those markers are not frequently used in Western countries where HCC is also often being diagnosed at more advanced stages. Thus, this score has not been evaluated on patients from Western countries.

\section{CUPI score}

The CUPI for HCC was identified on the basis of a cohort of 926 Chinese patients, most of them with hepatitis B virus (HBV) related cirrhosis ${ }^{[9]}$. CUPI combines the conventional TNM system and a number of other factors of liver function and tumor load (serum bilirubin, ascites, ALP, serum AFP and asymptomatic disease on presentation) [Table 7]. Patients are subsequently divided into three groups (lowrisk, intermediate-risk and high-risk) according to the sum of the weights of the six prognostic factors. The median survival for the low-risk, intermediate-risk, and high-risk groups were 10.1 months, 3.7 months and 1.4 months, respectively. The authors estimate that this classification has better estimation of survival than CLIP score and Okuda stage, although its discriminatory power in early stages is questionable, as the best 1-year survival was around 50\%. In 2011, the group validated the CUPI system in another cohort of 595 HCC patients with predominant $\mathrm{HBV}$ infection ${ }^{[59]}$. 
Table 7. Chinese University Prognostic Index ${ }^{[9]}$

\begin{tabular}{lc}
\hline & Scores \\
\hline TNM stage & -3 \\
I and II & -1 \\
IIIa and IIIb & 0 \\
IVa and IVb & -4 \\
Asymptomatic disease on presentation & 3 \\
Ascites & 2 \\
Alpha-fetoprotein $(\geq 500 \mathrm{ng} / \mathrm{mL})$ & \\
Total bilirubin $(\mu \mathrm{mol} / \mathrm{L})$ & 0 \\
$<34$ & 3 \\
$34-51$ & 4 \\
$\geq 52$ & 3 \\
Alkaline phosphatase $(\geq 200$ units/L) &
\end{tabular}

The prognostic factors used in this system are readily available in daily clinical practice and the score is determined based on the estimated Cox regression coefficient. However, CUPI was derived from a cohort of HCC patients primarily with HBV infection (79\% of the whole cohort). Thus, this system may not be suitable for application in Western populations with predominant HCV infection or a history of alcohol abuse. Another criticism levelled at CUPI was that only a small proportion of early-stage HCC patients have received surgery (10.4\%). Most of the patients in this cohort were in late stage and received only supportive care $(58.4 \%)^{[9]}$. Therefore, this system may not be preferred for assessing patients who undergo curative treatment, such as surgical resection or radiofrequency ablation (RFA). In comparison to other staging systems, the CUPI has not shown a prognostic advantage over other systems and has failed to gain widespread acceptance and usage. Moreover, though it is mainly used in Asian populations with a background of hepatitis B, still there is no evidence that CUPI has universal applicability among liver cancer patients of other races.

\section{Tokyo score}

Tokyo score was proposed by Tateishi et al. ${ }^{[11]}$ in 2005 based on a retrospective analysis of 403 HCC patients treated by percutaneous ablation at the University of Tokyo and was validated in 203 HCC patients who underwent surgery resection at the same institution. The main purpose of this study is to develop new prognostic scoring system for patients at early-stage who are candidates for radical therapy, such as percutaneous ablation or surgical resection. They used only serum albumin and bilirubin values as indicators of remnant liver function. This system consists of four factors: tumor size, number of tumor nodules, serum albumin and bilirubin which can be easily obtained from daily laboratory data or images before surgery [Table 8]. Scores are assigned to each of the four variables according to the estimated regression coefficient. Patients have total scores ranging from 0 to 6, Tokyo-0 patient having a better prognosis than those patients with Tokyo-6 (five-year survival rates of $78.7 \%$ vs. $14.3 \%$, respectively). In validation study, Tokyo staging system has shown to have a predictive ability equal to CLIP and better than BCLC classification ${ }^{[11]}$.

Tokyo score is useful in Japanese patients with early stage HCC requiring radical therapy but not suitable for use in patients with advanced stages of disease. Thus, its validation is required in Western population. Performance status and cancer-related symptoms have not been included in Tokyo score because most HCC patients in Japan were diagnosed at an early, asymptomatic stage of the disease due to nationwide screening program for viral hepatitis and surveillance in high-risk groups for HCC.

\section{Bilirubin-albumin-AFPL3-AFP-DCP score}

Bilirubin-albumin-AFPL3-AFP-DCP (BALAD) score is proposed by Toyoda et al ${ }^{[60]}$ in 2006 for the purpose of providing a simple and objective staging system that requires no imaging studies, pathological or clinical 
Table 8. Tokyo score ${ }^{[11]}$

\begin{tabular}{lc}
\hline Parameters & Scores \\
\hline Albumin $(\mathrm{g} / \mathrm{dL})$ & 0 \\
$>3.5$ & 1 \\
$2.8-3.5$ & 2 \\
$\quad<2.8$ & \\
Total bilirubin $(\mathrm{mg} / \mathrm{dL})$ & 0 \\
$\quad<1$ & 1 \\
$1-2$ & 2 \\
$>2$ & \\
Tumor size $(\mathrm{cm})$ & 0 \\
$<2$ & 1 \\
$2-5$ & 2 \\
$>5$ & \\
Tumor number & \\
$\leq 3$ & 0 \\
$>3$ & 2 \\
\hline
\end{tabular}

Table 9. BALAD score ${ }^{[60]}$

\begin{tabular}{|c|c|c|c|c|c|c|c|c|}
\hline & \multicolumn{3}{|c|}{ Bilirubin-albumin score } & & \multicolumn{4}{|c|}{ BALAD score } \\
\hline & 0 & 1 & 2 & & 0 & 1 & 2 & 3 \\
\hline Serum bilirubin $(\mathrm{mg} / \mathrm{dL})$ & $<1.0$ & $1.0-2.0$ & $>2.0$ & Bilirubin-albumin score ${ }^{\star}$ & A & $B$ & $\mathrm{C}$ & \\
\hline Serum albumin (g/dL) & $>3.5$ & $2.8-3.5$ & $<2.8$ & Number of elevated tumor markers & 0 & 1 & 2 & 3 \\
\hline
\end{tabular}

${ }^{\star}$ A: 0-1 points; B: 2-3 points; C: 4 points; BALAD: bilirubin-albumin-AFPL3-AFP-DCP

evaluations. This score is derived from the findings of a cohort of 2600 HCC patients treated at five Japanese institutions. BALAD scoring system is based on 5 serum markers: bilirubin and albumin as indicators of liver functional reserve, lens culinaris agglutinin reactive AFP-L3 $>15 \%$, AFP $>400 \mathrm{ng} / \mathrm{dL}$ and DCP $>$ $100 \mathrm{mAU} / \mathrm{mL}$ as factors reflecting tumor progression [Table 9]. Based on the sum of the scores assigned to these factors Japanese population of HCC could be stratified into six groups with distinct survivals. The discriminative ability of the BALAD score is comparable to that of the CLIP score and JIS score. The BALAD score has been validated in an independent cohort of Japanese population of HCC, as well as in the Caucasian population of HCC, and the score is consistently able to stratify the outcome of HCC into six group of patients with distinct median $\mathrm{OS}^{[5,66]}$. It has also been studied in a Chinese patient population of HBV related HCC and shown to have the corresponding median OS of BALAD scores of $1,2,3$ and 4 as $26.6,8.3,2.6$, and 1.9 months, respectively ${ }^{[62]}$. Although the BALAD score is a simple and objective tool for staging HCC it is not easy to measure the AFP-L3 and DCP values in routine clinical practice worldwide.

\section{ALPCS}

The ALPCS (Advanced Liver Cancer Prognostic System) was constructed by Yau et al. ${ }^{[48]}$ in 2008. It is derived from the analysis of a cohort of 1470 advanced HCC patients treated at a single center. To classify advanced HCC patients not indicated for surgical resection or locoregional therapy the authors identified 11 prognostic factors (ascites, abdominal pain, weight loss, Child-Pugh grade, ALP, serum total bilirubin, serum AFP, serum urea, tumor size, portal thrombosis and lung metastasis) using a multivariate Cox model and a point is given for each prognostic factor according to its statistical weight [Table 10]. Patients are subsequently divided into three prognostic groups (good: score $\leq 8$, intermediate: 9-15 and poor: $\geq 16$ ) [Table 11]. Survival curves for each prognostic group show clear differences, with a median OS of 7.9, 3.2 and 1.4 months for the good, intermediate and poor prognostic groups, respectively. The authors showed that the discriminatory ability of the ALPCS is significantly better than that of the Okuda system and CLIP score. However, the majority of patients included in this study (73\% of the whole cohort) were hepatitis B-related HCC. Therefore, ALPCS needs to be validated in a Western population with predominant HCV infection or alcohol abuse. In 
Table 10. Advanced liver cancer prognostic system ${ }^{[48]}$

\begin{tabular}{|c|c|c|c|c|c|c|}
\hline Parameters & 0 & 1 & 2 & 3 & 4 & 5 \\
\hline Ascites & No & & Yes & & & \\
\hline Abdominal pain & & No & & Yes & & \\
\hline Weight loss & No & & Yes & & & \\
\hline Child-Pugh & A & & B & & & C \\
\hline $\operatorname{ALP}(I U / L)$ & $\leq 200$ & & & $>200$ & & \\
\hline Total bilirubin (mg/dL) & & $\leq 2$ & $2-3$ & & $>3$ & \\
\hline Urea $(\mathrm{mmol} / \mathrm{L})$ & & $\leq 8.9$ & & $>8.9$ & & \\
\hline Portal vein thrombosis & & No & & & Yes & \\
\hline Tumor size & $\leq 5 \mathrm{~cm}$ & & & $>5 \mathrm{~cm}$ & & Diffuse \\
\hline Lung metastasis & & No & & & Yes & \\
\hline Alpha-fetoprotein $(\mathrm{ng} / \mathrm{mL})$ & $\leq 400$ & & & & $>400$ & \\
\hline
\end{tabular}

ALPCS: advanced liver cancer prognostic system; ALP: alkaline phosphates; IU: international unit

Table 11. Survival for each prognostic group of corresponding ALPCS score

\begin{tabular}{clc}
\hline \multicolumn{1}{c}{ Prognosis } & Score & 3 month survival \\
\hline Good (0-8) & $0-2$ & $>0.81$ \\
& $3-6$ & $0.72-0.8$ \\
Intermediate (9-15) & $7-8$ & $0.66-0.69$ \\
& 9 & 0.63 \\
& $10-12$ & $0.51-0.59$ \\
$13-14$ & $0.42-0.47$ \\
Poor (16-39) & 15 & 0.38 \\
& 16 & 0.33 \\
& $17-19$ & $0.21-0.29$ \\
& $20-22$ & $0.1-0.17$ \\
& $\geq 23$ & $<0.1$ \\
\hline
\end{tabular}

Table 12. Taipei Integrated System ${ }^{[63]}$

\begin{tabular}{llllc}
\hline & \multicolumn{4}{c}{ Scores } \\
\hline & 0 & 1 & 2 & 3 \\
Total tumor volume $\left(\mathrm{cm}^{3}\right)$ & $<50$ & $50-250$ & $250-500$ & $>500$ \\
Child-Pugh & A & B & C & \\
Alpha-fetoprotein $(\mathrm{ng} / \mathrm{mL})$ & $\leq 400$ & $>400$ & & \\
\hline
\end{tabular}

addition, considering 11 factors included into the system, calculation of the score is somewhat complicated in daily clinical practice.

\section{Taipei Integrated Score System score}

The Taipei Integrated Score System (TIS) was proposed by Hsu et al ${ }^{[63]}$ in 2010 . This system is derived from the study of a cohort of 2030 HCC patients undergoing different treatment modalities at a single institution in Taiwan. The authors included the total tumor volume (TTV) as an indicator of tumor burden and combined it with Child-Pugh grade (A, B and C: 0, 1 and 2 points, respectively) and AFP ( $<400 v s .>400 \mathrm{ng} / \mathrm{mL}: 0$ vs. 1 point) [Table 12]. Calculated TTV was categorized into four groups $\left(<50 \mathrm{~cm}^{3}, 50-250 \mathrm{~cm}^{3}, 250-500 \mathrm{~cm}^{3}\right.$ and $>$ $500 \mathrm{~cm}^{3}: 0,1,2$ and 3 points, respectively). The score identified six distinct prognostic groups. TIS shows superior prognostic value compared with the four current staging systems (CLIP, BCLC, JIS and Tokyo) for the whole cohort, independently of the treatment modality (curative or palliative). However, in a subgroup of 936 patients treated with curative intent, TIS failed to CLIP probably related to vascular invasion (a factor in the CLIP but not in the TIS) that was observed in $36.7 \%$ of the patients. Although the TTV based staging system is a useful and reliable system, it has some limitations. First, all tumors are not spherical. Therefore, TTV value may not be accurate in cases involving tumors that are infiltrative or numberless. Second, TIS 
Table 13. MESIAH Score ${ }^{[12]}$

\begin{aligned} \hline & MESIAH Score \\ \hline & $-0.232^{\star}$ (age in decades) \\ & $+0.099^{\star}($ MELD $) \\ & -0.391^{\star}$ (serum albumin level) \\ & $+0.290^{*}$ (tumor size) \\ & $+0.153^{\star}$ (tumor number) \\ & $+1.122^{*}$ (vascular invasion) \\ & $+1.130^{\star}$ (extrahepatic metastasis) \\ & $+0.082^{\star}$ (serum alpha-fetoprotein level) \\ & +1\end{aligned}

MESIAH: model to estimate survival in ambulatory HCC; MELD: model for end-stage liver disease

was constructed based on the results for a cohort of HCC patients with predominant HBV infection (HBV $51 \%$, HCV 27\%). Therefore, it needs to be externally validated in Western population.

\section{Model to estimate survival in ambulatory HCC patients score}

The model to estimate survival in ambulatory HCC patients score (MESIAH score) was developed by Yang et al.$^{[12]}$ from the Mayo group, in 2012. MESIAH score is derived from a cohort of 477 HCC patients treated at the Mayo Clinic [derivation cohort (DC)] and 904 HCC patients treated at a Korean institution [validation cohort (VC)]. Validation was done using a data set that is racially, geographically, chronologically and diagnostically disparate from the derivation set. The DC differed from VC with regard to the underlying liver disease $(\mathrm{DC}=\mathrm{HCV} 81 \%$ vs. $\mathrm{VC}=\mathrm{HBV} 75 \%)$ and treatment modality $(\mathrm{DC}=$ transplantation $31 \%$, resection $17 \%$, TACE $25 \% v s$. VC= resection 13\%, TACE 57\%). The authors identified independent predictors for survival in a multivariate Cox model [age, model for end-stage liver disease (MELD) score, serum albumin level, tumor size, tumor number, vascular invasion and extrahepatic metastasis], thus creating a new risk score [Table 13]. The authors include MELD as an indicator of liver disease severity. MELD has been shown to be a useful measure of hepatic insufficiency since it was adopted as a standard to determine organ allocation priorities among liver transplant candidates in the USA and elsewhere ${ }^{[64]}$, MELD is consisted of only laboratory variables (bilirubin, INR, creatinine) which are widely available and reproducible. The prognostic value of the MESIAH score was confirmed in the VC. The predictive accuracy of MESIAH is highly stable, irrespective of the underlying liver disease and/or treatment modality. More recently, the same group validated this score in another cohort of 1969 HCC patients with predominant HBV infection (74.6\%) treated at a Korean institution ${ }^{[65]}$. The discriminatory ability of the MESIAH score is better than that of the BCLC, CLIP, JIS and Tokyo. However, calculating the MESIAH score is somewhat complicated in daily clinical practice. Considering the advantages of superior predictive accuracy and objectivity and reproducibility of the prognostic factors, independent of the underlying liver disease and treatment modality, the MESIAH score is one of the most promising staging systems for evaluating HCC patients.

\section{Hong Kong Liver Cancer classification}

The Hong Kong Liver Cancer (HKLC) classification was developed by a Hong Kong group in $2014^{[4]}$. Like the BCLC, HKLC links HCC stages to treatment options. This system is based on four established prognostic factors: ECOG PS, Child-Pugh grade, liver tumor status and presence of extrahepatic vascular invasion or metastasis [Figure 2]. HKLC was derived from the results of a cohort of 3856 HCC patients primarily with HBV infection treated at single institution. Based on these prognostic factors, patients are classified in five main groups and nine subgroups with distinct survival outcomes. In the authors' analysis, HKLC classification exhibits better prognostic value than the BCLC classification. Regarding to problematic issues of BCLC such as heterogeneity of the stages B and C, and rigidity of treatment allocation, HKLC is able to better stratify patients in these stages into distinct groups with better survival outcomes based on more aggressive treatment recommendations than that observed in the BCLC treatment algorithm. Interestingly, 


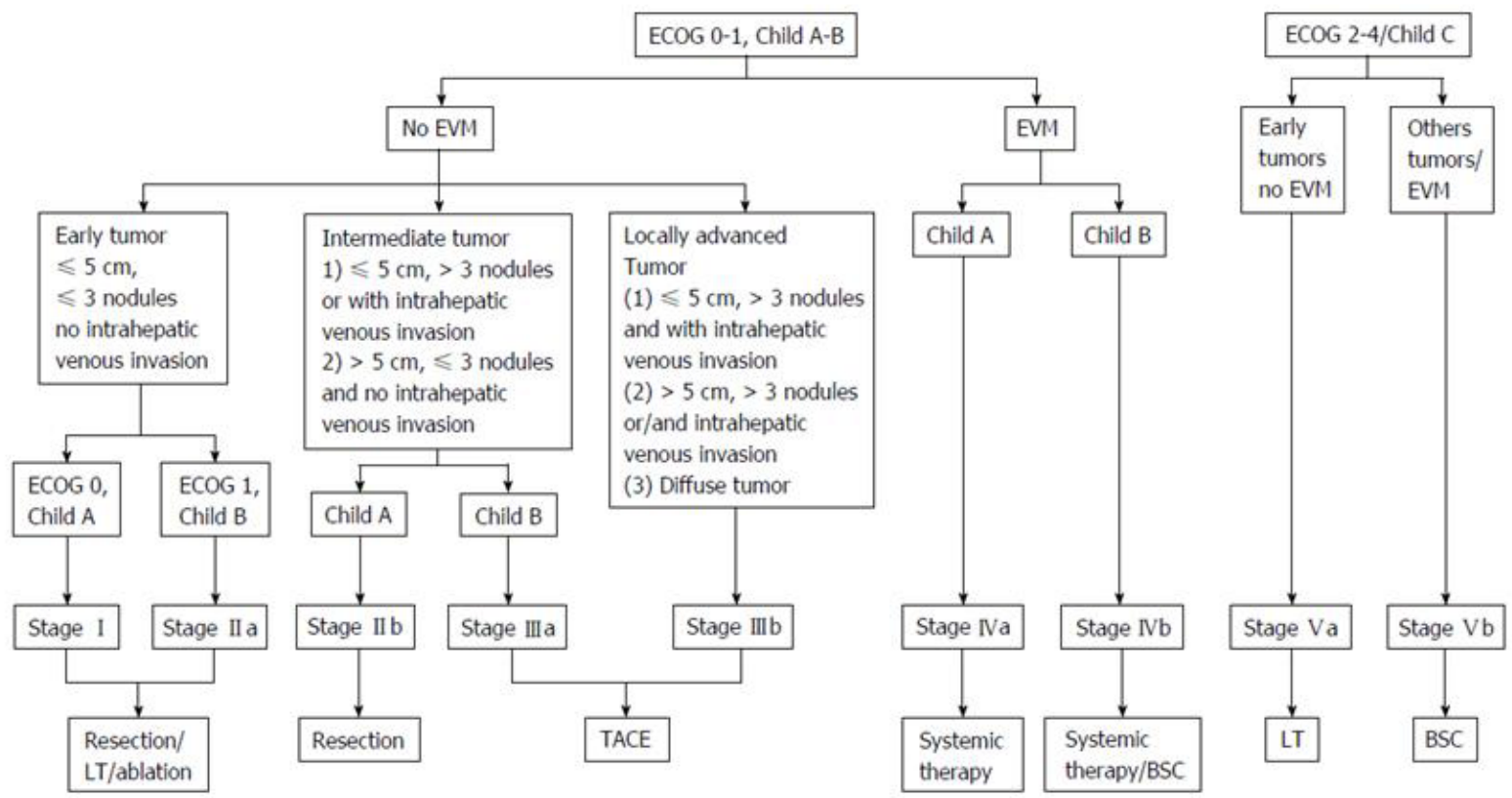

Figure 2. HKLC classification (from Adhoute et $a l^{[66]}$ Usefulness of staging systems and prognostic scores for hepatocellular carcinoma treatments). EVM: extrahepatic vascular invasion/metastasis; BSC: best supportive care; TACE: transarterial chemoembolization; ECOG: Eastern Cooperative Oncology Group

patients staged BCLC B and HKLC II had a survival probability of $52 \%$ at 5 years if they underwent surgical resection as first treatment, compared with a survival probability of $18.7 \%$ at 5 years if they received first-line TACE. This algorithm expands the scope of surgical resection. It offers expanded treatment recommendations such as surgical resection for BCLC B patients, TACE for BCLC C patients or LT for BCLC D patients which should be verified in a large-scale prospective study.

HKLC classification was derived from a cohort of HCC patients with predominant HBV infection (80\% of the whole cohort). Recently, the capability of HKLC in European cohorts have been challenged ${ }^{[66,67]}$. In a recent study, based on the pooled data from three European prospective cohort of 1693 patients with HCC, the BCLC staging system found to predict better in OS for European patients than the HKLC staging system $^{[67]}$. Twice more patients were eligible for a curative therapy with the HKLC algorithm, but its survival benefit remains to be investigated.

\section{ITA.LI.CA integrated staging system}

The ITA.LI.CA staging system was developed by Italian Liver Cancer Study group in 2016 for the purpose of providing a new prognostic system for HCC including both tumor staging to be used in the clinical management and an integrated prognostic score to predict patient survival ${ }^{[68]}$. This retrospective study was derived from the analysis of a cohort of over 5000 HCC patients from Italy (mainly HCV). External validation was performed using data from a Taiwanese cohort of over 2600 HCC patients (mainly HBV). Tumor staging $(0, A, B 1, B 2, B 3, C)$ is based on tumor characteristics such as largest tumor diameter, number of nodules, intra-and extrahepatic macroscopic vascular invasion, extrahepatic metastases [Table 14]. Multivariable survival model is then used to calculate the relative prognostic value of ITA.LI.CA tumor stage, ECOG performance status, Child-Pugh score (CPS), and AFP $>$ or $\leq 1,000 \mu \mathrm{g} / \mathrm{L}$ in predicting individual survival. Based on the model results, an ITA.LI.CA integrated prognostic score (from 0 to 13 points) is constructed [Table 15]. In the authors' analysis, the model had better discriminant ability than any of the existing staging systems (BCLC, CLIP, JIS, MESIAH and HKLC stage). 
Table 14. The ITA.LI.CA tumor staging system ${ }^{[68]}$

\begin{tabular}{lccc}
\hline Diameter of largest tumor $(\mathbf{c m})$ & Number of tumors & Vascular invasion or metastasis & Stage \\
\hline$\leq 2$ & 1 & No & 0 \\
$\leq 3$ & $2-3$ & No & A \\
$2-5$ & 1 & No & A \\
$3-5$ & $2-3$ & No & B1 \\
$>5$ & 1 & No & B1 \\
$>5$ & $2-3$ & No & B2 \\
$\leq 5$ & $>3$ & No & B2 \\
$>5$ & $>3$ & No & B3 \\
Any & Any & Intrahepatic & B3 \\
Any & Any & Extrahepatic & $\mathrm{C}$ \\
\hline
\end{tabular}

Table 15. Development of the ITA.LI.CA integrated prognostic score

\begin{tabular}{lcc}
\hline & Prognostic factor & Points \\
\hline ITA.LI.CA Tumor staging & 0 & 0 \\
& A & 1 \\
& B1 & 2 \\
& B2 & 3 \\
& B & 4 \\
ITA.LI.CA functional score & C & 5 \\
Child-Pugh score & 5 & \\
& 6 & 0 \\
& 7 & 1 \\
& 8 & 1 \\
ECOG PST & 9 & 2 \\
& $10-15$ & 2 \\
& 0 & 3 \\
AFP $(\mu g / L)$ & 1 & 0 \\
& 2 & 1 \\
\end{tabular}

ECOG PST: Eastern Cooperative Oncology Group performance status; AFP: alpha-fetoprotein

Some aspects of the ITA.LI.CA system are rooted in the BCLC staging system. Different than BCLC, ITA. LI.CA subclassifies BCLC stage B patients into B1, B2, and B3 categories based on degree of intrahepatic tumor burden and presence of intra-extrahepatic metastases. Finally, the serum biomarker AFP was incorporated as a surrogate for occult vascular invasion, distant metastases, or aggressive tumor biology. Although the model demonstrated good prognostic discrimination among study patients, it should be noted that there are significant differences in cancer etiology and treatment choices between European and Asian populations. Additionally, most patients in both cohorts had good performance status, compensated cirrhosis, and early or intermediate stage tumors. Whether ITA.LI.CA staging system would perform as well in cohorts with advanced tumor stage remains to be determined. Furthermore, it needs to be validated in prospective studies with more contemporary cohorts.

\section{Biomarkers}

Early diagnosis and treatment of HCC is crucial for achieving long term survival. Detection of tumor biomarkers is one of the main methods in reaching this goal. AFP is widely used as serum biomarker for HCC diagnosis, however, the diagnostic accuracy of HCC with serum AFP exhibits both sensitivity and specificity far below satisfaction, especially with small sizes of $\mathrm{HCC}^{[69]}$. With the development of new technology and advances in research, a number of new and specific biomarkers of HCC have been 
discovered. Besides AFP, Lens culinaris agglutinin-reactive AFP (AFP-L3) and DCP have been incorporated in current staging systems as factors reflecting tumor progression (BALAD and bm-JIS). Although some reports have not been consistent about the significance of both AFP-L3 and DCP in the diagnosis of HCC ${ }^{[70]}$, they are considered as promising biomarkers especially for the diagnosis of small HCC with low level of $\mathrm{AFP}^{[7]]}$. To improve their diagnostic sensitivity and specificity, combined tests of AFP, AFP-L3 and DCP are often applied in clinical practice ${ }^{[72]}$.

Vascular endothelial growth factor (VEGF), an endothelial cell mitogen that promotes neovascularization and endothelial cell proliferation, significantly increases in serum of HCC patients compared with control individuals and correlates with venous invasion, advanced tumor stage and poor prognosis ${ }^{[73,74]}$. Furthermore, the expression of VEGF in HCC tissues was related to invasiveness and metastasis of $\mathrm{HCC}^{[75]}$. However, VEGF may also be increased in other cancers and its value for early diagnosis of HCC is also unclear ${ }^{[76]}$. Additionally, other biomarkers such as Golgi glycoprotein 73, a transmembrane Golgi glycoprotein, and Glypican 3, a cell-surface heparan proteoglycan have been studied and found to be promising biomarkers which have high sensitivity and specificity for the diagnosis of small HCC with negative $\mathrm{AFP}^{[71]}$. With the development of genomics and proteomics, more and more new biomarkers will be discovered and used in clinical settings to diagnose different stages of HCC.

\section{CONCLUSION}

Over the past 20 years, diagnostic tools and treatment modalities have improved and screening programs have led to earlier diagnosis of HCC. Liver transplantation, hepatic resection, RFA, and transarterial chemoembolization have all been used in these patients to achieve a curative therapy. However, according to the degree of hepatic functional loss and heterogeneous nature of the tumor, optimal management for these patients remains controversial. Therefore, there is an increasing need for a staging system that can reflect the prognosis and permit the better stratification of these patients for clinical trials. To date, several staging systems have been proposed with various combinations of clinical, biochemical and pathological factors. However, search for a comprehensive staging system with appropriate treatment options applicable worldwide to all HCC patients despite geographical, financial and etiologic differences continues.

\section{DECLARATIONS}

\section{Authors' contributions}

The author contributed solely to the article.

\section{Availability of data and materials}

Not applicable.

\section{Financial support and sponsorship}

None.

\section{Conflicts of interest}

The author declared that there are no conflicts of interest.

\section{Ethical approval and consent to participate}

Not applicable.

\section{Consent for publication}

Not applicable. 


\section{Copyright}

(c) The Author(s) 2018.

\section{REFERENCES}

1. Bruix J, Llovet JM. Prognostic prediction and treatment strategy in hepatocellular carcinoma. Hepatology 2002;35:519-24

2. Marrero JA, Fontana RJ, Barrat A, Askari F, Conjeevaram HS, Su GL, Lok AS. Prognosis of hepatocellular carcinoma: comparison of 7 staging systems in an American cohort. Hepatology 2005;41:707-16.

3. Llovet JM, Brú C, Bruix J. Prognosis of hepatocellular carcinoma: the BCLC staging classification. Semin Liver Dis 1999;19:329-38.

4. Yau T, Tang VY, Yao TJ, Fan ST, Lo CM, Poon RT. Development of Hong Kong Liver Cancer staging system with treatment stratification for patients with hepatocellular carcinoma. Gastroenterology 2014;146:1691-700.e3.

5. Edge S, Byrd DR, Compton CC, Fritz AG, Greene FL, Trotti A. AJCC cancer staging manual. 7th ed. New York: Springer; 2010.

6. Okuda K, Ohtsuki T, Obata H, Tomimatsu M, Okazaki N, Hasegawa H, Nakajima Y, Ohnishi K. Natural history of hepatocellular carcinoma and prognosis in relation to treatment. Study of 850 patients. Cancer 1985;56:918-28.

7. A new prognostic system for hepatocellular carcinoma: a retrospective study of 435 patients: the Cancer of the Liver Italian Program (CLIP) investigators. Hepatology 1998;28:751-5.

8. Chevret S, Trinchet JC, Mathieu D, Rached AA, Beaugrand M, Chastang C. A new prognostic classification for predicting survival in patients with hepatocellular carcinoma. Groupe d'Etude et de Traitement du Carcinome Hepatocellulaire. J Hepatol 1999;31:133-41.

9. Leung TW, Tang AM, Zee B, Lau WY, Lai PB, Leung KL, Lau JT, Yu SC, Johnson PJ. Construction of the Chinese University Prognostic Index for hepatocellular carcinoma and comparison with the TNM staging system, the Okuda staging system, and the Cancer of the Liver Italian Program staging system: a study based on 926 patients. Cancer 2002;94:1760-9.

10. Kudo M, Chung H, Osaki Y. Prognostic staging system for hepatocellular carcinoma (CLIP score): its value and limitations, and a proposal for a new staging system, the Japan Integrated Staging Score (JIS score). J Gastroenterol 2003;38:207-15.

11. Tateishi R, Yoshida H, Shiina S, Imamura H, Hasegawa K, Teratani T, Obi S, Sato S, Koike Y, Fujishima T, Makuuchi M, Omata M. Proposal of a new prognostic model for hepatocellular carcinoma: an analysis of 403 patients. Gut 2005;54:419-25.

12. Yang JD, Kim WR, Park KW, Chaiteerakij R, Kim B, Sanderson SO, Larson JJ, Pedersen RA, Therneau TM, Gores GJ, Roberts LR, Park JW. Model to estimate survival in ambulatory patients with hepatocellular carcinoma. Hepatology 2012;56:614-21.

13. Schütte K, Schulz C, Link A, Malfertheiner P. Current biomarkers for hepatocellular carcinoma: surveillance, diagnosis and prediction of prognosis. World J Hepatol 2015;7:139-49.

14. Shouval D. HCC: what's the score? Gut 2002;50:749-50.

15. Cillo U, Bassanello M, Vitale A, Grigoletto FA, Burra P, Fagiuoli S, D’Amico F, Ciarleglio FA, Boccagni P, Brolese A, Zanus G, D’Amico DF. The critical issue of hepatocellular carcinoma prognostic classification: which is the best tool available? J Hepatol 2004;40:124-31.

16. Rabe C, Lenz M, Schmitz V, Pilz T, Fimmers R, Sauerbruch T, Caselmann WH. An independent evaluation of modern prognostic scores in a central European cohort of 120 patients with hepatocellular carcinoma. Eur J Gastroenterol Hepatol 2003;15:1305-15.

17. Giannini E, Risso D, Botta F, Romagnoli P, Malfatti F, Fumagalli A, Testa E, Podestà E, Chiarbonello B, Polegato S, Testa R. Prognosis of hepatocellular carcinoma in anti-HCV positive cirrhotic patients: a single-centre comparison amongst four different staging systems. J Intern Med 2004;255:399-408.

18. Ueno S, Tanabe G, Sako K, Hiwaki T, Hokotate H, Fukukura Y, Baba Y, Imamura Y, Aikou T. Discrimination value of the new western prognostic system (CLIP score) for hepatocellular carcinoma in 662 Japanese patients. Hepatology 2001;34:529-34.

19. Farinati F, Rinaldi M, Gianni S, Naccarato R. How should patients with hepatocellular carcinoma be staged? Validation of a new staging system. Cancer 2000;89:2266-73.

20. Prospective validation of the CLIP score: a new prognostic system for patients with cirrhosis and hepatocellular carcinoma. The Cancer of the Liver Italian Program (CLIP) Investigators. Hepatology 2000;31:840-5.

21. Levy I, Sherman M; Liver Cancer Study Group of the University of Toronto. Staging of hepatocellular carcinoma: assessment of the CLIP, Okuda, and Child-Pugh staging systems in a cohort of 257 patients in Toronto. Gut 2002;50:881-5.

22. Liu PH, Hsu CY, Hsia CY, Lee YH, Su CW, Huang YH, Lee FY, Lin HC, Huo TI. Prognosis of hepatocellular carcinoma: assessment of eleven staging systems. J Hepatol 2016;64:601-8.

23. Cillo U, Vitale A, Grigoletto F, Farinati F, Brolese A, Zanus G, Neri D, Boccagni P, Srsen N, D'Amico F, Ciarleglio FA, Bridda A, D'Amico DF. Prospective validation of the Barcelona Clinic Liver Cancer staging system. J Hepatol 2006;44:723-31

24. Marrero JA, Kudo M, Bronowicki JP. The challenge of prognosis and staging for hepatocellular carcinoma. Oncologist 2010;15 Suppl 4:2333.

25. Olthoff KM, Forner A, Hübscher S, Fung J. What is the best staging system for hepatocellular carcinoma in the setting of liver transplantation? Liver Transpl 2011;17 Suppl 2:S26-33.

26. Maida M, Orlando E, Cammà C, Cabibbo G. Staging systems of hepatocellular carcinoma: a review of literature. World J Gastroenterol 2014;20:4141-50.

27. Subramaniam S, Kelley RK, Venook Ap. A review of hepatocellular carcinoma (HCC) staging systems. Chin Clin Oncol 2013;2:33.

28. Llovet JM, Burroughs A, Bruix J. Hepatocellular carcinoma. Lancet 2003;362:1907-17.

29. European Association for the Study of the Liver; European Organisation for Research and Treatment of Cancer. EASLEORTC clinical practice guidelines: management of hepatocellular carcinoma. J Hepatol 2012;56:908-43.

30. Bruix J, Sherman M; American Association for the Study of Liver Diseases. Management of hepatocellular carcinoma: an update. Hepatology 2011;53:1020-2.

31. Oken MM, Creech RH, Tormey DC, Horton J, Davis TE, McFadden ET, Carbone PP. Toxicity and response criteria of the Eastern 
Cooperative Oncology Group. Am J Clin Oncol 1982;5:649-55.

32. Forner A, Llovet JM, Bruix J. Hepatocellular carcinoma. Lancet 2012;379:1245-55.

33. Befeler AS, Di Bisceglie AM. Hepatocellular carcinoma: diagnosis and treatment. Gastroenterology 2002;122:1609-19.

34. Huitzil-Melendez FD, Capanu M, O'Reilly EM, Duffy A, Gansukh B, Saltz LL, Abou-Alfa GK. Advanced hepatocellular carcinoma: which staging systems best predict prognosis? J Clin Oncol 2010;28:2889-95.

35. Zhang JF, Shu ZJ, Xie CY, Li Q, Jin XH, Gu W, Jiang FJ, Ling CQ. Prognosis of unresectable hepatocellular carcinoma: comparison of seven staging systems (TNM, Okuda, BCLC, CLIP, CUPI, JIS, CIS) in a Chinese cohort. PLoS One 2014;9:e88182.

36. Guglielmi A, Ruzzenente A, Pachera S, Valdegamberi A, Sandri M, D’Onofrio M, Iacono C. Comparison of seven staging systems in cirrhotic patients with hepatocellular carcinoma in a cohort of patients who underwent radiofrequency ablation with complete response. Am J Gastroenterol 2008;103:597-604.

37. Kim BK, Kim SU, Park JY, Kim DY, Ahn SH, Park MS, Kim EH, Seong J, Lee DY, Han KH. Applicability of BCLC stage for prognostic stratification in comparison with other staging systems: single centre experience from long-term clinical outcomes of 1717 treatment-naive patients with hepatocellular carcinoma. Liver Int 2012;32:1120-7.

38. Bolondi L, Burroughs A, Dufour JF, Galle PR, Mazzaferro V, Piscaglia F, Raoul JL, Sangro B. Heterogeneity of patients with intermediate (BCLC B) hepatocellular carcinoma: proposal for a subclassification to facilitate treatment decisions. Semin Liver Dis 2012;32:348-59.

39. Chang WT, Kao WY, Chau GY, Su CW, Lei HJ, Wu JC, Hsia CY, Lui WY, King KL, Lee SD. Hepatic resection can provide long-term survival of patients with non-early-stage hepatocellular carcinoma: extending the indication for resection? Surgery 2012;152:809-20.

40. Torzilli G, Belghiti J, Kokudo N, Takayama T, Capussotti L, Nuzzo G, Vauthey JN, Choti MA, De Santibanes E, Donadon M, Morenghi E, Makuuchi M. A snapshot of the effective indications and results of surgery for hepatocellular carcinoma in tertiary referral centers: is it adherent to the EASL/AASLD recommendations? An observational study of the HCC East-West study group. Ann Surg 2013;257:929-37.

41. Vitale A, Burra P, Frigo AC, Trevisani F, Farinati F, Spolverato G, Volk M, Giannini EG, Ciccarese F, Piscaglia F, Rapaccini GL, Di Marco M, Caturelli E, Zoli M, Borzio F, Cabibbo G, Felder M, Gasbarrini A, Sacco R, Foschi FG, Missale G, Morisco F, Svegliati Baroni G, Virdone R, Cillo U; Italian Liver Cancer (ITA.LI.CA) group. Survival benefit of liver resection for patients with hepatocellular carcinoma across different Barcelona Clinic Liver Cancer stages: a multicentre study; Italian Liver Cancer (ITA.LI.CA) group. J Hepatol 2015;62:61724.

42. Xu L, Peng ZW, Chen MS, Shi M, Zhang YJ, Guo RP, Lin XJ, Lau WY. Prognostic nomogram for patients with unresectable hepatocellular carcinoma after transcatheter arterial chemoembolization. J Hepatol 2015;63:122-30.

43. Xue TC, Xie XY, Zhang L, Yin X, Zhang BH, Ren ZG. Transarterial chemoembolization for hepatocellular carcinoma with portal vein tumor thrombus: a meta-analysis. BMC Gastroenterol 2013;13:60.

44. Korean Liver Cancer Study Group (KLCSG); National Cancer Center, Korea (NCC). 2014 KLCSG-NCC Korea practice guideline for the management of hepatocellular carcinoma. Gut Liver 2015;9:267-317.

45. Hsu CY, Lee YH, Hsia CY, Huang YH, Su CW, Lin HC, Lee RC, Chiou YY, Lee FY, Huo TI. Performance status in patients with hepatocellular carcinoma: determinants, prognostic impact, and ability to improve the Barcelona Clinic Liver Cancer system. Hepatology 2013;57:112-9.

46. Huitzil-Melendez FD, Capanu M, O'Reilly EM, Duffy A, Gansukh B, Saltz LL, Abou-Alfa GK. Advanced hepatocellular carcinoma: which staging systems best predict prognosis? J Clin Oncol 2010;28:2889-95.

47. Collette S, Bonnetain F, Paoletti X, Doffoel M, Bouché O, Raoul JL, Rougier P, Masskouri F, Bedenne L, Barbare JC. Prognosis of advanced hepatocellular carcinoma: comparison of three staging systems in two French clinical trials. Ann Oncol 2008;19:1117-26.

48. Yau T, Yao TJ, Chan P, Ng K, Fan ST, Poon RT. A new prognostic score system in patients with advanced hepatocellular carcinoma not amendable to locoregional therapy: implication for patient selection in systemic therapy trials. Cancer 2008;113:2742-51

49. Marsh JW, Dvorchik I, Bonham CA, Iwatsuki S. Is the pathologic TNM staging system for patients with hepatoma predictive of outcome? Cancer 2000;88:538-43.

50. Llovet JM, Bruix J, Fuster J, Castells A, Garcia-Valdecasas JC, Grande L, Franca A, Brú C, Navasa M, Ayuso MC, Solé M, Real MI, Vilana R, Rimola A, Visa J, Rodés J. Liver transplantation for small hepatocellular carcinoma: the tumor-node-metastasis classification does not have prognostic power. Hepatology 1998;27:1572-7.

51. Vauthey JN, Lauwers GY, Esnaola NF, Do KA, Belghiti J, Mirza N, Curley SA, Ellis LM, Regimbeau JM, Rashid A, Cleary KR, Nagorney DM. Simplified staging for hepatocellular carcinoma. J Clin Oncol 2002;20:1527-36.

52. Durand F, Regimbeau JM, Belghiti J, Sauvanet A, Vilgrain V, Terris B, Moutardier V, Farges O, Valla D. Assessment of the benefits and risks of percutaneous biopsy before surgical resection of hepatocellular carcinoma. J Hepatol 2001;35:254-58.

53. Chun YH, Kim SU, Park JY, Kim DY, Han KH, Chon CY, Kim BK, Choi GH, Kim KS, Choi JS, Ahn SH. Prognostic value of the 7th edition of the AJCC staging system as a clinical staging system in patients with hepatocellular carcinoma. Eur J Cancer 2011;47:2568-75.

54. Makuuchi M, Belghiti J, Belli G, Fan ST, Lau JW, Ringe B, Strasberg SM, Vauthey JN, Yamaoka Y, Yamasaki S; Working Group of the International Scientific Committee of the International Hepato-Pancreato-Biliary Association. IHPBA concordant classification of primary liver cancer: working group report. J Hepatobiliary Pancreat Surg 2003;10:26-30.

55. Kudo M, Chung H, Haji S, Osaki Y, Oka H, Seki T, Kasugai H, Sasaki Y, Matsunaga T. Validation of a new prognostic staging system for hepatocellular carcinoma: the JIS score compared with the CLIP score. Hepatology 2004;40:1396-405.

56. Toyoda H, Kumada T, Kiriyama S, Sone Y, Tanikawa M, Hisanaga Y, Yamaguchi A, Isogai M, Kaneoka Y, Washizu J. Comparison of the usefulness of three staging systems for hepatocellular carcinoma (CLIP, BCLC, and JIS) in Japan. Am J Gastroenterol 2005;100:1764-71.

57. Ikai I, Takayasu K, Omata M, Okita K, Nakanuma Y, Matsuyama Y, Makuuchi M, Kojiro M, Ichida T, Arii S, Yamaoka Y; Liver Cancer Study Group of Japan. A modified Japan Integrated Stage score for prognostic assessment in patients with hepatocellular carcinoma. J Gastroenterol 2006;41:884-92.

58. Kitai S, Kudo M, Minami Y, Ueshima K, Chung H, Hagiwara S, Inoue T, Ishikawa E, Takahashi S, Asakuma Y, Haji S, Osaki Y, Oka H, Seki T, Kasugai H, Sasaki Y, Matsunaga T. A new prognostic staging system for hepatocellular carcinoma: value of the biomarker combined 
Japan integrated staging score. Intervirology 2008;51 Suppl 1:86-94.

59. Chan SL, Mo FK, Johnson PJ, Liem GS, Chan TC, Poon MC, Ma BB, Leung TW, Lai PB, Chan AT, Mok TS, Yeo W. Prospective validation of the Chinese University Prognostic Index and comparison with other staging systems for hepatocellular carcinoma in an Asian population. J Gastroenterol Hepatol 2011;26:340-7.

60. Toyoda H, Kumada T, Osaki Y, Oka H, Urano F, Kudo M, Matsunaga T. Staging hepatocellular carcinoma by a novel scoring system (BALAD score) based on serum markers. Clin Gastroenterol Hepatol 2006;4:1528-36.

61. Fox R, Berhane S, Teng M, Cox T, Tada T, Toyoda H, Kumada T, Kagebayashi C, Satomura S, Johnson PJ. Biomarker-based prognosis in hepatocellular carcinoma: validation and extension of the BALAD model. Br J Cancer 2014;110:2090-8.

62. Chan SL, Mo F, Johnson P, Li L, Tang N, Loong H, Chan AW, Koh J, Chan AT, Yeo W. Applicability of BALAD score in prognostication of hepatitis B-related hepatocellular carcinoma. J Gastroenterol Hepatol 2015;30:1529-35.

63. Hsu CY, Huang YH, Hsia CY, Su CW, Lin HC, Loong CC, Chiou YY, Chiang JH, Lee PC, Huo TI, Lee SD. A new prognostic model for hepatocellular carcinoma based on total tumor volume: the Taipei Integrated Scoring System. J Hepatol 2010;53:108-17.

64. Kamath PS, Kim WR; Advanced Liver Disease Study Group. The model for end-stage liver disease (MELD). Hepatology 2007;45:797-805.

65. Kim BH, Park JW, Nam BH, Kwak HW, Kim WR. Validation of a model to estimate survival in ambulatory patients with hepatocellular carcinoma: a single-centre cohort study. Liver Int 2014;34:e317-23.

66. Adhoute X, Penaranda G, Bronowicki JP, Raoul JL. Usefulness of the HKLC vs. the BCLC staging system in a European HCC cohort. J Hepatol 2015;62:492-3.

67. Kolly P, Reeves H, Sangro B, Knöpfli M, Candinas D, Dufour JF. Assessment of the Hong Kong Liver Cancer Staging System in Europe. Liver Int 2016;36:911-7.

68. Farinati F, Vitale A, Spolverato G, Pawlik TM, Huo TL, Lee YH, Frigo AC, Giacomin A, Giannini EG, Ciccarese F, Piscaglia F, Rapaccini GL, Di Marco M, Caturelli E, Zoli M, Borzio F, Cabibbo G, Felder M, Sacco R, Morisco F, Biasini E, Foschi FG, Gasbarrini A, Svegliati Baroni G, Virdone R, Masotto A, Trevisani F, Cillo U; ITA.LI.CA study group. Development and validation of a new prognostic system for patients with hepatocellular carcinoma. PLoS Med 2016;13:e1002006.

69. Daniele B, Bencivenga A, Megna AS, Tinessa V. Alpha-fetoprotein and ultrasonography screening for hepatocellular carcinoma. Gastroenterology 2004;127:S108-12.

70. Yi X, Yu S, Bao Y. Alpha-fetoprotein-L3 in hepatocellular carcinoma: a meta-analysis. Clin Chim Acta 2013;425:212-20.

71. Zhao Y, Wang M, Cui C, Zhang L, Liao F, Li H, Wu X. Significance of combined tests of serum golgi glycoprotein 73 and other biomarkers in diagnosis of small primary hepatocellular carcinoma. Cancer Biomark 2015;15:677-83.

72. Caviglia GP, Abate ML, Petrini E, Gaia S, Rizzetto M, Smedile A. Highly sensitive alpha-fetoprotein, lens culinaris agglutinin-reactive fraction of alpha-fetoprotein and des-gamma-carboxyprothrombin for hepatocellular carcinoma detection. Hepatol Res 2016;46:E130-5.

73. Poon RT, Ho JW, Tong CS, Lau C, Ng IO, Fan ST. Prognostic significance of serum vascular endothelial growth factor and endostatin in patients with hepatocellular carcinoma. Br J Surg 2004;91:1354-60.

74. Zhan P, Qian Q, Yu LK. Serum VEGF level is associated with the outcome of patients with hepatocellular carcinoma: a metaanalysis. Hepatobiliary Surg Nutr 2013;2:209-15.

75. Yuen MF, Lai CL.Serological markers of liver cancer. Best Pract Res Clin Gastroenterol 2005;19:91-9.

76. Hu J, Xu Y, Shen ZZ, Wang Z, Lu Q, Yang GH, Ding ZB, Fan J, Zhou J. High expressions of vascular endothelial growth factor and platelet-derived endothelial cell growth factor predict poor prognosis in alpha-fetoprotein-negative hepatocellular carcinoma patients after curative resection. J Cancer Res Clin Oncol 2009;135:1359-67. 